\title{
The Effects of Physical Exercise on Cognition: How Heart Rate Variability Can Predict Cognitive Performances
}

OPEN ACCESS

Edited by:

Victor Hugo C. de Albuquerque, University of Fortaleza, Brazil

Reviewed by:

Nizar Bouguila

Concordia University, Canada M. Shamim Hossain King Saud University, Saudi Arabia Antonino Galletta University of Messina, Italy

*Correspondence: Giovanna Sannino giovanna.sannin

Specialty section: This article was submitted to

Brain-Computer Interfaces,

a section of the journal Frontiers in Human Neuroscience

Received: 19 June 2020

Accepted: 15 July 2020 Published: 31 August 2020

Citation: Sannino G, De Falco I, De Pietro G and Stranges S (2020) The Effects of Physical Exercise on Cognition: How Heart Rate Variability Can Predict

Cognitive Performances.

Front. Hum. Neurosci. 14:312. doi: 10.3389/fnhum.2020.00312

\begin{abstract}
Giovanna Sannino $^{1 *}$, Ivanoe De Falco ${ }^{1}$, Giuseppe De Pietro ${ }^{1}$ and Saverio Stranges ${ }^{2,3,4}$
${ }^{1}$ ICAR, National Research Council of Italy, Naples, Italy, ${ }^{2}$ Department of Epidemiology and Biostatistics, Schulich School of Medicine and Dentistry, Western University, London, ON, Canada, ${ }^{3}$ Department of Family Medicine, Schulich School of Medicine and Dentistry, Western University, London, ON, Canada, ${ }^{4}$ Department of Population Health, Luxembourg Institute of Health, Strassen, Luxembourg
\end{abstract}

Objectives: Investigate and identify the relationship between physical exercise and cognitive performance measured by using different cognitive tests taken from Cambridge Brain Science (CBS).

Methods: Thirty subjects, divided into two groups (aerobic and effort), undergo twelve cognitive tests from CBS. A comparison between the pre-and post-exercise results in terms of cognitive performance differences is carried out. Regression analysis between Heart Rate Variability (HRV) features and CBS tests results is performed.

Results: In most CBS tests, there is an improvement, or at least a confirmation, of the subject's cognitive ability, for both groups. Reasoning (80-100\%), concentration (80-87\%), and planning tests (93-100\%) seem to undergo critical positive changes. The regression analysis, performedby using a set of different algorithms, has demonstrated that it is possible, by monitoring the HRV during the exercise, to predict to some extent the cognitive performance, i.e., the CBS tests results. The best performing regression algorithms are Simple Linear (Quade Test-aerobic group: 2.098, effort group: 3.350, both groups: 2.747) and REPTree (Quade Test-aerobic group: 2.955, effort group: 3.315, lboth groups: 3.121). The statistical analysis has proved that physical activity is statistically useful for the subjects in improving their cognitive performance.

Conclusions: This study has numerically appraised the improvement, the conservation, or the worsening on different aspects of cognition. The found mathematical relationship between physical exercise and cognitive performance suggests that it is possible to predict the beneficial effect of various exercises on executive and attentive control.

Keywords: cognition, physical exercise, heart rate variability, prediction of cognitive performances, pilot study

\section{INTRODUCTION}

The importance of exercise to overall well-being is documented in the scientific literature. Regular exercise is essential for sustaining a healthy body weight and can help in the maintenance of normal blood lipid levels and blood pressure (Fletcher et al., 1992). The benefits of exercise extend beyond physiological changes; exercise is also advantageous to mental health, having been shown to improve self-esteem and self-confidence and to reduce symptoms of depression (Fletcher et al., 1992). The helpfulness of exercise is so systematic and profound that it is recommended in the prevention and management of various medical conditions, including cardiovascular disease, 
metabolic syndrome, osteoporosis, a number of neoplastic diseases, and various mental illnesses, such as anxiety (Fletcher et al., 1992). However, an often-overlooked aspect of exercise is the effect it has on cognition and the brain.

In fact, there is increasing evidence that exercise can have drastic effects on almost all aspects of brain health, including improved learning and memory, reduced symptoms of depression, better outcomes associated with brain injury, and delayed onset and magnitude of cognitive decline associated with various neurodegenerative diseases (Cotman et al., 2007).

Literature shows more and more papers aiming at linking physical activity to improvements in brain function and cognition, as for example (Luft et al., 2009; Paterson and Warburton, 2010; Ahlskog et al., 2011; Erickson et al., 2011; Luque-Casado et al., 2013; Blondell et al., 2014; Scherder et al., 2014; Bherer, 2015; Prakash et al., 2015; Tsunoda et al., 2015; Bauman et al., 2016; Tivadar, 2017). Research carried out on animals shows the positive effect of enriched environments, including access to exercise equipment (such as running wheels) on neuronal growth. The same effect is shown when neural systems involved in learning and memory are considered. This indicates that physically active behaviors have an effect both on the cognitive function and on the related brain structures (Vaynman and Gomez-Pinilla, 2006). Similar results have been found when research is carried out on humans (Booth and Lees, 2006), with recent research in neuroimaging techniques showing that exercise yields apparent modifications both in structure and in the function of the brain.

However, none of the mentioned studies gives the possibility to predict the cognitive performance of the subjects. This possibility is what we investigate in this paper, and represents its novelty.

We present a study aiming to investigate the impact on, and the mathematical relationship between, physical activity and cognitive performances. These latter are measured by using a number of different cognitive tests taken from Cambridge Brain Science, the CBS tests 1 . The study, detailed in section 2, involves thirty subjects, divided into two groups, each with specific physical activity, and twelve cognitive tests.

As a result of this study, we have been able to create a brandnew data set linking physical activity and the results of the cognitive tests over time. To the best of our knowledge, this is the first data set of this kind, and no other freely available such data sets exist.

Working on this data set, we have conducted different kinds of analysis in section 3. In detail, as described in section 3.1, we have first evaluated how the physical activity impacts on cognitive activities such as reasoning, work memory, and verbal skills. Or rather, we have numerically appraised, both before and after the physical training, the improvement, the conservation, or the worsening on different aspects of cognition, each of them represented by a CBS test. This evaluation has been made in terms of cognitive performance by defining a suitable numerical $\Delta$ Score.

$\overline{1_{\text {www.cambridgebrainsciences.com }}}$
Additionally, as detailed in section 3.2, we have analyzed if a mathematical relationship exists between physical exercise and cognitive performance. This has been done by studying if a regression model can be found that is able to predict the cognitive performance, i.e., the CBS test scores, by using the Heart Rate Variability (HRV) features monitored during the physical exercise. To perform regression, several algorithms are considered, and the best six, in terms of three different regression quality indexes, are reported.

We have chosen to use the HRV, a non-invasive parameter, because this allows measuring the dynamic autonomic responses during exercise through the analysis of the ElectroCardiogram (ECG) signal, i.e., the HRV, as demonstrated in important studies in the scientific literature (Makivić et al., 2013; Ngandu et al., 2015). These results indicate that it is possible to use the HRV parameters for the analysis of the stress experienced by the body during the training phase, and for better understanding the physiological recovery following the training. As a matter of fact, it is sensible to hypothesize that modifications in the Autonomic Nervous System (ANS) patterns, yepresented by modifications in the HRV, might act as helpful parâmeters for the management of both physical fatigue and exercise intensity

The purpose of finding a mathematical relationship between $\mathrm{HRV}$ and cognitive performance is to predict the beneficial effect of various exercise programs (aerobics or muscle strength) on executive and attentive control (work memory, distributed focus, inhibition of response, interference on control).

This is a particularly relevant research activity aiming to demonstrate if a direct correlation between the trend of HRV and cognitive performance exists. In fact, if demonstrated, it would possible to design prevention activities based on personalized exercise programs that, using HRV analysis, are optimized for the improvement of cognitive abilities.

Finally, as mentioned in section 3.3, a statistical analysis is run to: (i) understand if the physical activity is statistically useful for the subjects in improving their performance at solving the 12 CBS tests; and (ii) to evaluate the quality of the regression models.

\section{METHODS}

\subsection{Ethics Statement}

This study has been conducted in compliance with the World Medical Association Declaration of Helsinki on Ethical Principles for Medical Research Involving Human Subjects (World Medical Association, 2008).

The protocol guidelines of the study have been written in conformity with the SPIRIT 2013 Statement (Chan et al., 2013), and, before the start of the experimental session, the participants read and signed an informed consent statement. Suitable information was given to them about their right to quit the experiment whenever they wanted, and each of the subjects was informed in a detailed way about the goal of this study when the experimental session ended. Finally, all the data from all the participants were examined, filed, and described in a completely anonymous form. 


\subsection{The Recruitment: Participants and Procedure for the Data Acquisition}

We recruited 30 healthy participants (age range: $18-48$ years old), of which 18 males and 12 females.

In order to be considered suitable for the trial process, the subjects had to comply with these criteria:

- Adults aged over 18 years;

- Subjects with no level of physical fitness as defined in the normative values of the American College of Sports Medicine (Sharp, 1993);

- Subjects being no professional athletes;

- No people suffering from any pathological cardiovascular conditions, neurological or psychiatric disorders or other severe diseases.

The procedure of the study has been outlined in Figure 1.

In the following, we report a brief summary of the phases of the protocol performed for data collection:

Day 1 :

- The participant is informed about the purposes of the study and how it will be conducted. Information material is provided, and the participant is invited to sign the informed consent.

- The participant is apprised of the use of the wearable device that she/he should wear during physical activity for the continuous acquisition of the Heart Rate (HR) data, the Zephyr BioHarness BH3 (Zephyr Technology, 2016). Together with the device, detailed instructions are given on how to place the sensor and on how to use it.

- The participant is informed about the 12 cognitive tests of the CBS that they will have to perform after each physical exercise, and about the modality for the on-line compilation through the platform on $\mathrm{CBS}$ site 2 .

Figure 2 shows the 12 cognitive tests used: the green ones are related to the Short Term Memory (STM) cognition, those relating to the reasoning abilities are shown in blue, those relating to verbal skills in red, whereas the purple ones make reference to memory.

- The participant is informed about the scheduling of the activities, which is set as follows:

Task 1 - Initial cognitive assessment: day 1;

Task 2 - 1st battery of 12 cognitive tests: day 5 ;

Task 3 - 2nd battery of 12 cognitive tests: day 10;

Task 4 - 3rd battery of 12 cognitive tests: day 15;

Task 5 - 4th battery of 12 cognitive tests: day 20;

Task 6 - 5th battery of 12 cognitive tests: day 25;

Task 7 - Final cognitive assessment: day 30.
Between one task and another, the participant is invited to perform a minimum of $3 \mathrm{~h}$ of physical exercise (even noncontinuative), preferably $1 \mathrm{~h}$ per day for 3 days. The type of physical exercise to be performed will depend on the target group to which it will be assigned, which can be either aerobic or effort. If assigned to the aerobic group, the subject must perform a type of aerobic exercise, such as running or cycling. If, instead, the subject is assigned to the effort group, she/he must perform a type of exercise that is short but intense, such as lifting weights, bending, or lifting at the bar. Of the 30 people recruited, 15 were chosen in a random way and allocated to the effort group (Group 1), while the other 15 to the aerobic group (Group 2).

- The initial cognitive assessment is requested on site.

Days 5, 10, 15, 20, and 25 :

- The participant is asked to send the KR data acquired until now;

- The results of each battery of 12 cognitive tests in each day are collected through the CBS platform.

Day 30 :

- The participant is asked to send the HR data acquired so far;

- The final cognitive assessment is requested on site;

- The participant will return the instrumentation received.

\subsection{The Cognitive Measurements}

The current study uses CBS tests ${ }^{3}$ to assess cognitive performance. CBS is a set of 12 on-line tests used to measure different aspects of cognitive function. This collection of tests assesses aspects of inhibition, selective attention, reasoning, verbal STM, spatial working memory, planning, and cognitive flexibility. Together, these tests collectively and comprehensively assess three cognitive domains: STM, reasoning, and verbal abilities, based on behavioral data (from over 44,000 individuals) and neuroimaging studies that have demonstrated that each domain is supported by a separate brain network (Hampshire et al., 2012). They have been validated in patients (Owen et al., 1990, 1991, 1993) and healthy populations (Owen et al., 1996a,b) and have been shown to be sensitive to subtle cognitive changes due to neurodegeneration (Owen et al., 1992, 1993) or pharmacological intervention (Lange et al., 1992; Mehta et al., 2000). In addition, the tests are designed to be engaging to maximize participant compliance. Completion of the battery requires between 35 and $45 \mathrm{~min}$, which is faster and more convenient than many other pen-and-paper neuropsychological batteries. Taken together, the CBS battery is ideal to use in the current study because it is easy and fast to complete, can be used to assess even the most subtle changes in cognition, and the categorization of tests into latent cognitive domains will help

$\overline{{ }^{2} \text { www.cambridgebrainsciences.com }}$

$\overline{{ }^{3} \text { www.cambridgebrainsciences.com }}$ 


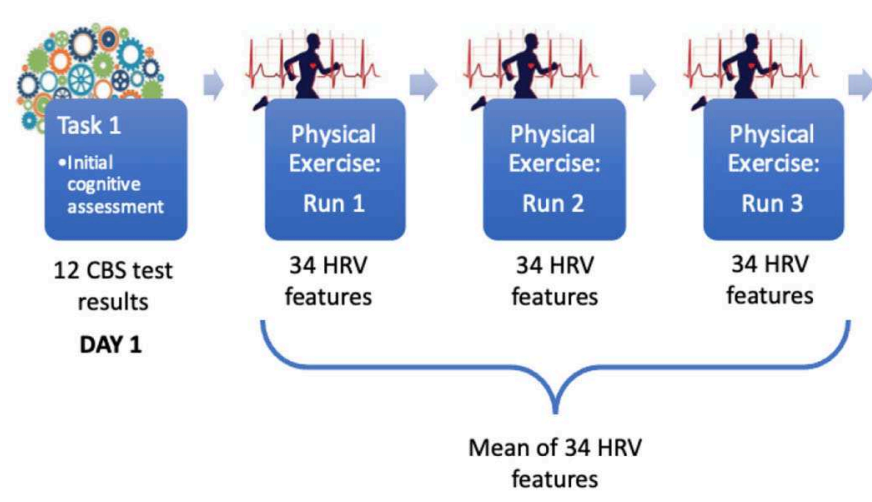

features
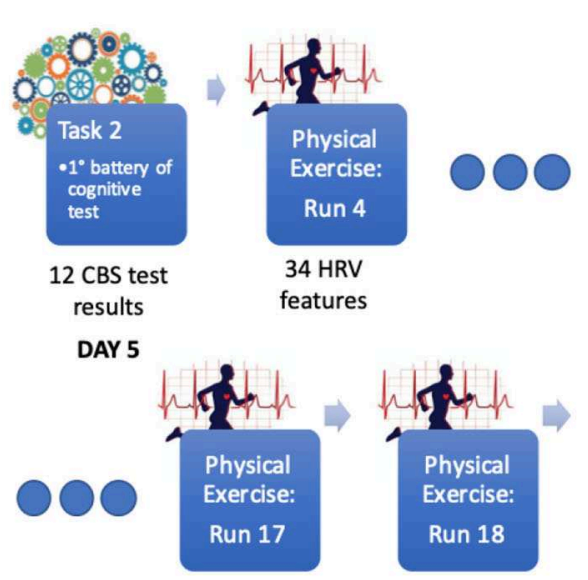

34 HRV features

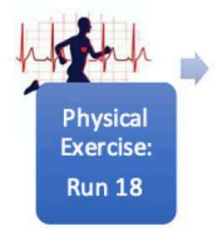

34 HRV features

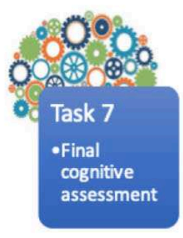

12 CBS test results

FIGURE 1 | Schema of the protocol performed for the data collection phase.

to provide an objective definition of exactly which cognitive processes are influenced by physical activity.

Below we give the summary of the CBS tests used in this study, and the cognitive area they are related to.

- Memory: Monkey Ladder, Spatial Span, Paired Associates, Digit Span;

- Reasoning: Odd One Out, Grammatical Reasoning;

- Concentration: Spatial Interlocking Polygons;

- Planning: Spatial Planning, Token Search.

\subsection{The HRV Measurements}

The HRV measurements have been extracted from the HR data monitored by using the Zephyr BioHarness BH3 Sensor (Zephyr Technology, 2016). This HR sensor can be used at the gym, on a run, while exercising at home, or during physical therapy. It is small, wearable, and compatible with many Bluetooth devices, and very suitable for personal and clinical use.

All HR data were processed using a Matlab-based software, named Kubios (Tarvainen et al., 2014), designed at the University of Kuopio, Finland. This is a state-of-the-art tool for the extraction and the analysis of the HRV characteristics from the HR data. Through Kubios, a set of parameters was extracted in the Time domain, in the Frequency domain, and in the NonLinear domain.

Before extracting the HRV parameters, each record is cleaned from power line interferences, muscle and movement artifacts, through the use of a cubic spline interpolation procedure (Daskalov and Christov, 1997; Mateo and Laguna, 2000).
The RR- interval, also called the NN-interval, is determined by the distance between two successive heartbeats in a normal rhythm of the ECG signal. As interference may take place between artifacts in the RR interval time series and the analysis of these signals, we decided to use the Kubios Artifacts removal RR filter set to a maximum threshold of 5\%.

After filtering, the analysis of the signals took place. In detail, 34 characteristic parameters were extracted, summarized in Table 1.

The features from 1 to 9 are related to the Time domain, the features from 10 to 22 refer to the Frequency domain, while those from 24 to 34 are related to the Non-Linear domain.

These characteristics were extracted from each HR data acquisition related to each run linked to a single exercise session of each participant.

\subsection{The Database}

The average values of the HRV parameters relative to the runs between two successive batteries of $12 \mathrm{CBS}$ tests have been correlated with the CBS tests results of the second of these batteries.

In practice, referring to Figure 1, for each individual exercise session (Run), a vector of $34 \mathrm{HRV}$ features has been extracted. The average of the HRV characteristics relative to the runs between two successive batteries of 12 CBS tests is then computed.

To better understand the construction mechanism of each database item, let us suppose that the subject performed three exercise runs between day 1 , in which she/he underwent the initial cognitive assessment, and day 5, when she/he engages in the first battery of CBS tests. From each HR signal monitored in each of the three exercise runs, $34 \mathrm{HRV}$ features are extracted. So, for each HRV feature, we have three values, one for each exercise run. We have computed the average of these three values. The 


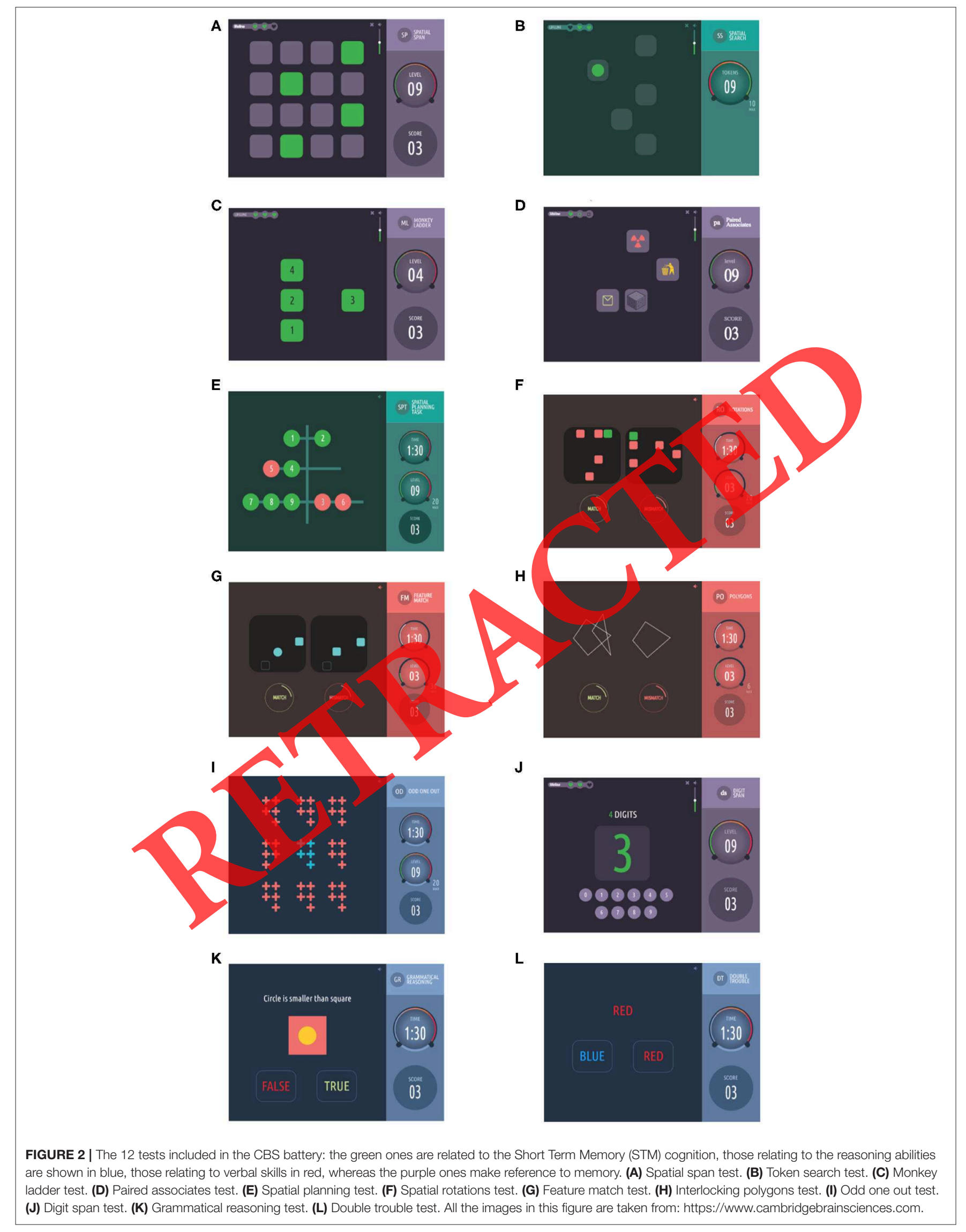




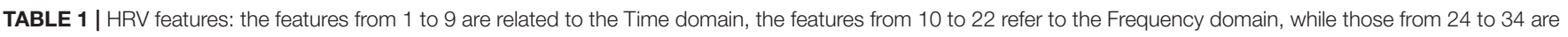
related to the non-linear domain.

\begin{tabular}{|c|c|c|c|}
\hline & Measure & Unit & Description \\
\hline 1 & MeanRR & $\mathrm{ms}$ & The mean of RR intervals \\
\hline 2 & STDRR & ms & Standard deviation of RR (NN) intervals \\
\hline 3 & MeanHR & $1 / \min$ & The mean of HRs values \\
\hline 4 & STDHR & $1 / \min$ & Standard deviation of HRs values \\
\hline 5 & RMSSD & ms & Square root of the mean squared differences between successive RR intervals \\
\hline 6 & NN50 & count & Number of successive RR interval pairs that differ more than $50 \mathrm{~m}$ \\
\hline 7 & pNN50 & $\%$ & NN50 divided by the total number of RR intervals \\
\hline 8 & RRtri & & The integral of the RR interval histogram divided by the height of the histogram \\
\hline 9 & TINN & Ms & Baseline width of the RR interval histogram \\
\hline 10 & PeakVLF & $\mathrm{Hz}$ & Peak frequencies of the very-low-frequency band \\
\hline 11 & PeakLF & $\mathrm{Hz}$ & Peak frequency of the Low Frequency (LF) band $(0.04-0.15 \mathrm{~Hz})$ \\
\hline 12 & PeakHF & $\mathrm{Hz}$ & Peak frequency of the High Frequency (HF) band $(0.15-0.4 \mathrm{~Hz})$ \\
\hline 13 & VLF & $m s^{2}$ & Absolute power of the very-low-frequency band $(0.0033-0.04 \mathrm{~Hz})$ \\
\hline 14 & LF & $\mathrm{ms}^{2}$ & Absolute powers of LF band, calculated with \\
\hline 15 & $\mathrm{HF}$ & $\mathrm{ms}^{2}$ & Absolute powers of HF band, calculated wi \\
\hline 16 & Relative VLF & $\%$ & Relative power of the very-low-frequen \\
\hline 17 & Relative LF & $\%$ & Relative power of the low-fre \\
\hline 18 & Relative HF & $\%$ & Relative power of the hic \\
\hline 19 & $\begin{array}{l}\text { Norm. Power } \\
\text { LF }\end{array}$ & nu & Relative power of the \\
\hline 20 & $\begin{array}{l}\text { Norm. Power } \\
\text { HF }\end{array}$ & nu & Relative power \\
\hline 21 & Total Power & $\mathrm{ms}^{2}$ & Sum of the energy in the VLF, LF, and HF bands \\
\hline 22 & LF/HF & $\%$ & Ratio between LF and HF band powers \\
\hline 23 & SD1 & $\mathrm{ms}$ & Of the Poincare plot perpendicular to the line of identity \\
\hline 24 & $\mathrm{SD} 2$ & $\mathrm{~ms}$ & è plot along to the line of identity \\
\hline 25 & ApEn & & v \\
\hline 26 & SampEn & & Sample entropy \\
\hline 27 & D2 & & Correlation Dimension \\
\hline 28 & DFA1 & & Short-term fluctuation slope in Detrended Fluctuation Analysis \\
\hline 29 & DFA2 & & Long-term fluctuation slope in Detrended Fluctuation Analysis \\
\hline 30 & RPLmean & & Recurrent Plot $(\mathrm{RP})$ mean line length \\
\hline 31 & RPLmax & & RP maximum line length \\
\hline 32 & RPREC & & RP Recurrence Rate \\
\hline 33 & & $\%$ & RP Determinism \\
\hline 34 & & & RP Shannon Entropy \\
\hline
\end{tabular}

Following this procedure, for each subject, we have obtained a number of six items. Each of these latter is constituted as below:

$$
i t e m=i d ; \operatorname{avg}\left(\left.\overrightarrow{f(H R V)}\right|_{1} ^{34}\right) ;\left.\overrightarrow{f(C B S)}\right|_{1} ^{12}
$$

where:

- $i d$ : is a value from 1 to 30 that unequivocally and anonymously identifies each participant;

- $\operatorname{avg}\left(\left.\overrightarrow{f(H R V)}\right|_{1} ^{34}\right)$ : is a vector containing the average values of the $34 \mathrm{HRV}$ features computed over the runs performed within two successive batteries of 12 CBS tests;

As a result of all the pre-processing phases and of the procedure faced, the data set used in this study consists of 180 items (6 items for 30 subjects), 90 of which related to group 1 (effort group), and 90 to group 2 (aerobic group).

\section{RESULTS}

\subsection{Impact of Physical Exercise on Cognitive Performance}

We have performed an analysis to determine whether the different exercise regimes (aerobic vs. effort exercise) had differential effects on cognition, and how they reflected in the 
change in performance over time. To this aim, for each subject, for the generic i-th CBS test (among the 12) we have defined:

$$
\Delta \text { Score }_{i} \%=\left.\frac{C B S_{i}(\text { day }=30)-C B S_{i}(\text { day }=1)}{\max (C B S)_{i}-\min (C B S)_{i}} * 100.0\right|_{i=1} ^{12}
$$

where

- $\max (C B S)_{i}$ : is the maximum among the values obtained by all the subjects over the i-th CBS test;

- $\min (C B S)_{i}$ : is the minimum among the values obtained by all the subjects over the i-th CBS test.

As shown in Figure 3, we can see that in some cases there has been an improvement in cognitive performance, whereas in others this is not the case. This improvement is different between the two groups, effort and aerobic.

To better understand how many subjects have achieved an improvement in cognition from the physical exercise, we report in Table 2 these numerical results expressed in percentage. The most significant percentages are reported in bold. As we can see, tests related to the reasoning, in particular Double Trouble and Grammatical Reasoning, have obtained a significant improvement. In fact, for the Double Trouble all the subjects of group $1(100 \%)$ have achieved better performance at the end of the study (day $=30$ ) with respect to the assessment made at the beginning of the study (day $=1)$. Meanwhile, for the Grammatical Reasoning, the number of cases in which an improvement has been registered is greater in Group 2 (aerobic), with respect to Group 1 (effort). Good improvements have also been registered for the concentration, especially in the Spatial Rotation test, in which, for both groups, we have an improvement in at least $80 \%$ of the subjects. Finally, also for the planning there is a significant improvement in almost all subjects of both groups, in detail in $100 \%$ of the subjects for Group 1 (effort) and in 93\% of the subjects for Group 2 (aerobic). No important consequences have been registered for the tests related to the memory in both groups. This means that physical exercise does not impact so much on memory performance.

\subsection{The Regression Analysis to Predict the Cognitive Performance}

From a hardware viewpoint, all the experiments reported in this paper have been effected on an iMac with High Sierra (Mac OsX 10.13.2) operating system, a 3.6-GHz Intel Core i7 processor, 8GB DDR4 at 2,400 MHz, and a 1 TB hard disk.

We have performed our experiments in the Waikato Environment for Knowledge Analysis (WEKA), version 3.8.1 (Garner, 1995). In it, we have taken advantage of a wide set of regression models coming from artificial intelligence, some already contained in the basic WEKA version, and some others freely downloadable from its repository. We have executed a good number of such models, and we have chosen to report here the best-performing algorithms, i.e., the ones which have achieved the best numerical results in our experiments. These have resulted in being the Support
Vector Machine (SVM) (Zeng et al., 2008; Bouguila and Amayri, 2009), the Multi-Layer Perceptron (MLP) Artificial Neural Network (Rumelhart et al., 1988), the InstanceBased K-nearest neighbors (IBk) (Aha et al., 1991), the Reduced Error Pruning Tree (REPTree) (Breslow and Aha, 1997), the Simple Linear and the Additive regression (Stone, 1985).

We have run all the regression models over the data set by performing five-fold cross-validation. This protects us from the overfitting problem by dividing the data set into five folds and estimating regression quality on each fold.

It is important to underline here that we chose not to carry out any parameter tuning phase that could assist us in finding the most appropriate values for the parameters of the different regression algorithms. Also, we chose not to rely on Principal Component Analysis, rather we have made use for the regression of all the $34 \mathrm{HRV}$ features extracted from the signals.

Considering that the two types of physical activity impact differently on the same characteristics of HRV, the analysis has been separately performed for each group. Therefore, 12 regression models were identified, each one to predict a single CBS test result for the effort group (Group 1), and other 12 regression models were identified for the aerobic group (Group 2).

To evaluate the regression models used in this study, we have chosen three quality indicators, the Pearson correlation coefficient $(\rho)$, the Mean Absolute Error (MAE), and the Root Mean Square Error (RMSE).

Correlation is a bivariate analysis measuring the strength of association between two variables as well as the direction of this relationship. Given a pair of random variables $y$ and $\hat{y}$, where $y_{i}$ is the actual output and $\hat{y}_{i}$ is the model's prediction, $\rho$ is defined as:

$$
\rho_{y, \hat{y}}=\frac{\operatorname{cov}(y, \hat{y})}{\sigma_{y} \sigma_{\hat{y}}}
$$

where:

- $\operatorname{cov}$ : is the covariance between $y$ and $\hat{y}$;

- $\sigma_{y}$ : is the standard deviation of $y$;

- $\sigma_{\hat{y}}$ : is the standard deviation of $\hat{y}$

As concerns the strength of the relationship, the value of $\rho$ ranges within -1 and +1 , and the higher the value, the better the model. When the value of $\rho$ lies around \pm 1 , a perfect degree of association, be it direct or inverse, is said to exist between the two variables.

In MAE, the error is computed as the average of the absolute differences between the target values and the corresponding predictions. Mathematically, it is calculated using the formula:

$$
M A E=(1 / n) \sum_{i=1}^{n}\left|y_{i}-\hat{y}_{i}\right|
$$

It is a linear score, so all the individual differences are assigned equal weights. As an example, the difference between 30 and 20 is considered as twice that between 30 and 25 . 


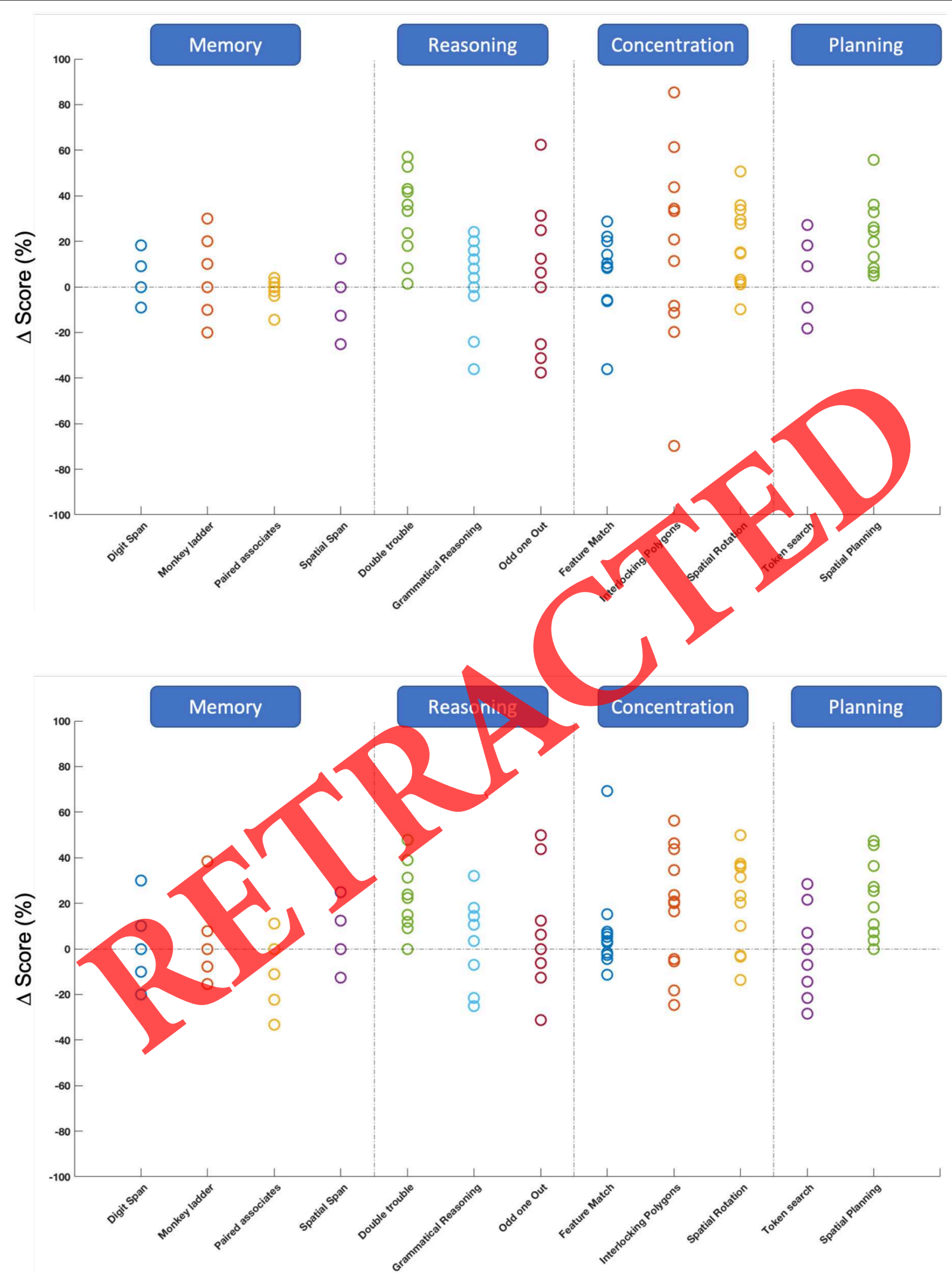

FIGURE 3 | Upper pane: $\Delta$ Scores of each subject belonging to the Group 1 (Effort group) calculated for the 12 CBS tests. Lower pane: $\Delta S c o r e s$ of each subject belonging to the Group 2 (Aerobic group) calculated for the 12 CBS tests. 
TABLE 2 | Percentages of subjects who have achieved an improvement, a conservation, or a worsening on the CBS tests results.

\begin{tabular}{|c|c|c|c|c|c|c|}
\hline & \multicolumn{3}{|c|}{ Group 1} & \multicolumn{3}{|c|}{ Group 2} \\
\hline & $\Delta>0$ & $\Delta=0$ & $\Delta<0$ & $\Delta>0$ & $\Delta=0$ & $\Delta<0$ \\
\hline Digit span & $27 \%$ & $53 \%$ & $20 \%$ & $47 \%$ & $13 \%$ & $40 \%$ \\
\hline Monkey ladder & $33 \%$ & $27 \%$ & $40 \%$ & $33 \%$ & $33 \%$ & $33 \%$ \\
\hline Paired association & $27 \%$ & $20 \%$ & $53 \%$ & $13 \%$ & $40 \%$ & $47 \%$ \\
\hline Spatial span & $47 \%$ & $20 \%$ & $33 \%$ & $53 \%$ & $13 \%$ & $33 \%$ \\
\hline Double trouble & $100 \%$ & $0 \%$ & $0 \%$ & $93 \%$ & $7 \%$ & $0 \%$ \\
\hline Gram. reasoning & $60 \%$ & $7 \%$ & $33 \%$ & $80 \%$ & $0 \%$ & $20 \%$ \\
\hline Odd one out & $60 \%$ & $7 \%$ & $33 \%$ & $47 \%$ & $13 \%$ & $40 \%$ \\
\hline Features match & $80 \%$ & $0 \%$ & $20 \%$ & $67 \%$ & $0 \%$ & $33 \%$ \\
\hline Interlocking polygons & $67 \%$ & $0 \%$ & $33 \%$ & $67 \%$ & $0 \%$ & $33 \%$ \\
\hline Spatial rotation & $87 \%$ & $0 \%$ & $13 \%$ & $80 \%$ & $0 \%$ & $20 \%$ \\
\hline Token search & $47 \%$ & $0 \%$ & $53 \%$ & $47 \%$ & $20 \%$ & $33 \%$ \\
\hline Spatial planning & $100 \%$ & $0 \%$ & $0 \%$ & $93 \%$ & $7 \%$ & $0 \%$ \\
\hline
\end{tabular}

$\Delta$ Refers to the $\Delta$ Score defined in Equation (2).

The most significant percentages are reported in bold.

The RMSE is defined by the equation:

$$
R M S E=\sqrt{(1 / n) \cdot \sum_{i=1}^{n}\left(y_{i}-\hat{y}_{i}\right)^{2}}
$$

The lower are the values for MAE and RMSE, the better the model is.

As said above, among all the models tested, we report the results achieved by the best six models, namely SVM, MLP, REPTree, IBk, Simple Linear, and Additive Regression. The numerical results, obtained over the train and test sets, are shown in the tables from Tables 3-5 in terms of $\rho$, MAE, and RMSE specified in the equations above. In each of these tables, we show in bold the best values achieved oyer each CBS test for both train and test sets.

Over the train set, IBk is, without doubt, the best algorithm. As a matter of fact, for MAE and RMSE it achieves perfect regression, with no error. Also for $p$ it often reaches the highest possible final value, i.e., 1.0. On the other hand, the outcomes achieved over the test set present a totally different scenario. This difference in IBk results for the two sets proves that this algorithm is highly afflicted by overfitting. This term is used to express that the IBk regression model achieved is customized to the train set elements, so it faces problems when it has to deal with the never-before-seen test set instances.

\subsection{Statistical Analysis}

Two important issues arise from the results shown above.

The first issue takes into account the results from a cognitive viewpoint. Namely, it raises questions about the usefulness of the physical activity: has this latter statistically helped those subjects improve their performance at solving the 12 tests? If the answer is positive, which kind of activity, effort or aerobic, has been more helpful? And, is it the same for all the tests?
The second issue, instead, concerns the quality of the regression results obtained. Namely, from a statistical viewpoint, which is the best algorithm, from among the six shown here, for each of the two kinds of activity? And, is the same algorithm the best in both cases?

For both issues, we have performed a suitable statistical analysis. Showing the whole set of related tables and findings would result in a too-high number of tables, which would be beyond the limit allowed by the journal. Hence, we show here a summary about our analysis. Interested readers may find in the below-mentioned papers a thorough description of the complete statistical analysis procedures followed here.

For the first issue, the Wilcoxon signed-rank test (Derrac et al., 2011) has been used. The choice of the Wilcoxon signed-rank test comes from the fact that here we wish to deal with repeated measurements over a same set of subjects, hence this test is wellsuited. The most important reason here to prefer Wilcoxon to the $t$-test is that it is known that, whenever the sample sizes are small, say less than 30 , the results of $t$-test can become little reliable. In this paper, we have small sets of samples, namely 15 subjects for each class and each test, so this is exactly the case. Moreover, unlike the $t$-test, for Wilcoxon there is no need to assume a normal distribution, and for it the outliers are of lower impact on the results than they are in t-test.

The results of this test are reported in Table 6 and have shown that both kinds of physical activity are useful to improve cognitive performance. Namely, the aerobic activity of Group 1 (effort) has turned out to be statistically helpful for both tests related to planning, whereas the effort activity of subjects in Group 2 (aerobic) has proven statistically useful for all the three tests related to concentration. Finally, when all the subjects in both groups are considered together, all the three tests linked to concentration provide statistically superior results at the end of the study than they did at the beginning.

For the second issue, instead, as we have reported the results of six different algorithms over a total of 12 tests, the use 
TABLE 3 | Numerical results, obtained over train and test sets, related to $\rho$.

\begin{tabular}{|c|c|c|c|c|c|c|c|c|c|c|c|c|}
\hline \multicolumn{13}{|c|}{ Group 1} \\
\hline & \multicolumn{2}{|c|}{ SVM } & \multicolumn{2}{|c|}{ MLP } & \multicolumn{2}{|c|}{ REPTree } & \multicolumn{2}{|c|}{ IBk } & \multicolumn{2}{|c|}{ Simple linear } & \multicolumn{2}{|c|}{ Additive } \\
\hline & Train & Test & Train & Test & Train & Test & Train & Test & Train & Test & Train & Test \\
\hline Digit span & 0.822 & -0.368 & 0.957 & 0.025 & 0.805 & 0.323 & 0.979 & 0.121 & 0.445 & 0.370 & 1.000 & -0.251 \\
\hline Monkey ladder & 0.772 & 0.672 & 1.000 & 0.593 & 0.917 & 0.449 & 0.956 & 0.632 & 0.445 & 0.730 & 0.998 & 0.225 \\
\hline Paired associates & 0.756 & 0.514 & 0.937 & -0.205 & 0.000 & 0.000 & 0.953 & -0.267 & 0.533 & 0.169 & 0.961 & -0.119 \\
\hline Spatial span & 0.627 & -0.115 & 0.955 & -0.127 & 0.955 & -0.127 & 1.000 & -0.292 & 0.307 & -0.249 & 1.000 & 0.135 \\
\hline Double trouble & 0.810 & 0.275 & 1.000 & 0.239 & 0.000 & 0.000 & 0.986 & -0.533 & 0.403 & 0.760 & 1.000 & 0.774 \\
\hline Grammatical reasoning & 0.734 & 0.632 & 1.000 & 0.517 & 0.509 & 0.290 & 1.000 & 0.223 & 0.520 & 0.856 & 0.975 & 0.292 \\
\hline Odd one out & 0.869 & 0.346 & 1.000 & -0.123 & 0.000 & 0.000 & 0.966 & 0.127 & 0.459 & 0.538 & 1.000 & 0.174 \\
\hline Feature match & 0.800 & 0.304 & 1.000 & 0.007 & 0.503 & 0.517 & 1.000 & 0.701 & 0.466 & 0.535 & 0.994 & 0.493 \\
\hline Interlocking polygons & 0.885 & 0.003 & 0.969 & -0.085 & 0.738 & -0.419 & 1.000 & -0.276 & 0.501 & 0.042 & 1.000 & 0.102 \\
\hline Spatial planning & 0.627 & -0.115 & 0.955 & -0.127 & 0.000 & 0.000 & 1.000 & -0.292 & 0.307 & -0.249 & 1.000 & 0.135 \\
\hline Token search & 0.686 & -0.098 & 1.000 & 0.131 & 0.000 & 0.000 & 1.000 & -0.042 & 0.486 & 0.166 & 0.961 & 0.092 \\
\hline Spatial rotations & 0.759 & -0.080 & 0.984 & 0.045 & 0.000 & 0.000 & 0.975 & 0.222 & 0.410 & & 1.000 & -0.749 \\
\hline \multicolumn{13}{|c|}{ Group 2} \\
\hline & Train & Test & Train & Test & Train & Test & Train & Test & & Test & Train & Test \\
\hline Digit span & 0.743 & -0.114 & 0.969 & -0.118 & 0.000 & 0.000 & 1.000 & -0.123 & & & 1.000 & -0.151 \\
\hline Monkey ladder & 0.736 & 0.263 & 0.996 & 0.299 & 0.000 & 0.000 & 1.000 & & & & 0.932 & -0.325 \\
\hline Paired associates & 0.286 & 0.070 & 0.971 & 0.511 & 0.000 & 0.000 & 0.969 & & & & 1.000 & 0.392 \\
\hline Spatial span & 0.746 & 0.047 & 1.000 & 0.256 & 0.576 & 0.092 & 1.000 & & & & 0.915 & 0.009 \\
\hline Double trouble & 0.749 & 0.151 & 0.974 & 0.460 & 0.000 & 0.000 & 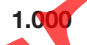 & & & 0.126 & 0.966 & 0.095 \\
\hline Grammatical reasoning & 0.651 & -0.111 & 0.990 & 0.276 & 0.850 & 0.147 & & & 0.390 & 0.090 & 0.991 & -0.170 \\
\hline Odd one out & 0.791 & -0.373 & 0.949 & -0.197 & 0.000 & 0.0 & 0.987 & & 0.490 & -0.302 & 0.971 & 0.010 \\
\hline Feature match & 0.601 & 0.607 & 1.000 & 0.204 & 0.000 & 0.0 & 0.979 & 0.296 & 0.314 & 0.179 & 0.982 & -0.258 \\
\hline Interlocking polygons & 0.704 & 0.093 & 1.000 & 0.044 & 0.64 & 0.0 & 1.000 & 0.343 & 0.378 & -0.137 & 0.938 & 0.103 \\
\hline Spatial planning & 0.746 & 0.047 & 1.000 & -0.256 & 0.57 & & & -0.121 & 0.473 & 0.144 & 0.915 & 0.009 \\
\hline Token search & 0.736 & 0.397 & 1.000 & -0.425 & 0.000 & & 0.985 & 0.145 & 0.298 & -0.131 & 0.961 & 0.354 \\
\hline Spatial rotations & 0.780 & 0.024 & 0.987 & 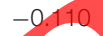 & 0.485 & & 1.000 & -0.076 & 0.390 & -0.345 & 0.954 & -0.079 \\
\hline
\end{tabular}

The values in bold are the best values achieved over each CBS test for both train and test sets

of non-parametrical statistical analysis is needed. By following (Demsar, 2006; Derrac et al., 2011), we have used Quade test, that takes account for the fact that not all the problems are equally difficult, or that the results obtained by the algorithms over them show larger differences, as it is the case here for the 12 cognitive tests. We have considered all the three quality indicators and all the subjects involved in this trial, and we have performed both the Quade statistical test and the related post-hoc procedures. The results over Group 1 (effort), shown in Tables 7, 8, reveal that Simple Linear is statistically better than the other five algorithms, followed by REPTree.

In the last row of Table 7, the value of the test statistic is also shown. This is a parameter specific to the particular statistical test executed. For the Quade test used in this paper, the test statistic is distributed according to an F-distribution with two degrees of freedom given by (number of algorithms - 1), and by (number of problems -1$)^{\star}$ (number of algorithms - 1), respectively. In this case, we have six algorithms and 12 problems (the 12 cognitive tests), so their values are equal to 5 and 175 .

In the same row of the table, the corresponding $p$-value is shown. It represents the smallest level of significance that results in the rejection of the null hypothesis. This latter is a statement of no difference among the tested algorithms. Such a $p$-value is computed through the statistics of the test considered: low values strongly suggest the existence of significant differences among the algorithms considered.

As concerns Table 8, instead, the row at its bottom reports for each post-hoc procedure the threshold value Th. This is important because the procedure under account rejects those equivalence hypotheses characterized by an adjusted $p$ value lower than or equal to Th. Moreover, $\mathrm{p}$ represents the unadjusted $p$-value for the algorithm under account, as contrasted to the adjusted $p$-values resulting from the various post-hoc procedures used. Also, the variable $\mathrm{z}$ is the test statistic for this comparison, and its definition can be found in Derrac et al. (2011).

Over Group 2 (aerobic), instead, the results reported in Tables 9, 10 reveal that SVM turns out to be statistically better, REPTree being the runner up.

Given that we have obtained different conclusions when working with the two groups separately, the question arises about which the best algorithm is when all the subjects in the two groups are considered at the same time. Based on the results of the Quade statistical test shown in Tables 11, 12, the bestperforming algorithm is, in this case, Simple Linear, REPTree being the runner-up. 
TABLE 4 | Numerical results, obtained over train and test sets, related to MAE.

\begin{tabular}{|c|c|c|c|c|c|c|c|c|c|c|c|c|}
\hline \multicolumn{13}{|c|}{ Group 1} \\
\hline & \multicolumn{2}{|c|}{ SVM } & \multicolumn{2}{|c|}{ MLP } & \multicolumn{2}{|c|}{ REPTree } & \multicolumn{2}{|c|}{ IBk } & \multicolumn{2}{|c|}{ Simple linear } & \multicolumn{2}{|c|}{ Additive } \\
\hline & Train & Test & Train & Test & Train & Test & Train & Test & Train & Test & Train & Test \\
\hline Digit span & 0.482 & 1.763 & 0.158 & 1.865 & 0.828 & 1.310 & 0.000 & 1.564 & 1.228 & 1.257 & 0.162 & 1.553 \\
\hline Monkey ladder & 0.355 & 0.407 & 0.005 & 0.645 & 0.260 & 0.576 & 0.000 & 0.446 & 0.611 & 0.346 & 0.019 & 0.938 \\
\hline Paired associates & 0.387 & 0.595 & 0.243 & 0.932 & 0.716 & 0.669 & 0.000 & 1.199 & 0.635 & 0.784 & 0.071 & 1.053 \\
\hline Spatial span & 0.511 & 0.772 & 0.016 & 0.842 & 0.016 & 0.842 & 0.000 & 1.002 & 0.770 & 0.766 & 0.102 & 1.120 \\
\hline Double trouble & 6.212 & 14.371 & 3.939 & 18.727 & 14.379 & 9.334 & 0.000 & 14.583 & 14.013 & 7.378 & 2.250 & 7.845 \\
\hline Grammatical reasoning & 2.062 & 4.065 & 0.065 & 5.734 & 3.055 & 5.630 & 0.000 & 6.340 & 4.227 & 3.446 & 0.471 & 6.346 \\
\hline Odd one out & 1.159 & 3.163 & 0.031 & 5.037 & 3.158 & 2.802 & 0.000 & 3.961 & 2.530 & 2.603 & 0.447 & 3.299 \\
\hline Feature match & 12.623 & 40.143 & 0.851 & 61.627 & 21.039 & 32.286 & 0.000 & 37.289 & 22.212 & 41.637 & 3.716 & 40.893 \\
\hline Interlocking polygons & 5.118 & 83.118 & 0.714 & 41.409 & 12.719 & 26.773 & 0.000 & 30.596 & 16.250 & 26.687 & 3.146 & 18.189 \\
\hline Spatial planning & 0.511 & 0.772 & 0.016 & 0.842 & 0.808 & 0.633 & 0.000 & 1.002 & 0.770 & 0.766 & 0.102 & 1.120 \\
\hline Token search & 0.601 & 1.879 & 0.007 & 1.899 & 1.193 & 1.550 & 0.000 & 1.682 & 1.041 & 1.767 & 0.196 & 1.952 \\
\hline Spatial rotations & 13.929 & 54.789 & 10.577 & 86.243 & 27.419 & 30.149 & 0.000 & 40.039 & 25.341 & & 3.796 & 48.654 \\
\hline \multicolumn{13}{|c|}{ Group 2} \\
\hline & Train & Test & Train & Test & Train & Test & Train & Test & & & Train & Test \\
\hline Digit span & 1.634 & 1.546 & 1.215 & 3.919 & 2.740 & 1.214 & 0.000 & & & & 0.422 & 1.374 \\
\hline Monkey ladder & 0.603 & 1.050 & 0.391 & 1.516 & 1.195 & 0.825 & 0.000 & & & & 0.362 & 1.389 \\
\hline Paired associates & 2.644 & 1.225 & 0.969 & 4.178 & 4.027 & 2.416 & 0.000 & & & & 1.791 & 3.874 \\
\hline Spatial span & 0.357 & 2.593 & 0.028 & 3.046 & 0.652 & 2.478 & $0: 0$ & & & 2.372 & 0.240 & 2.466 \\
\hline Double trouble & 8.474 & 13.615 & 2.016 & 12.041 & 15.649 & 10. & & & 17038 & 16.447 & 3.580 & 16.928 \\
\hline Grammatical reasoning & 2.238 & 6.686 & 0.729 & 6.252 & 1.611 & & 0.000 & & 3.520 & 5.613 & 1.087 & 7.155 \\
\hline Odd one out & 1.061 & 3.401 & 0.217 & 4.667 & 2.308 & & 0.000 & 3.867 & 1.882 & 3.502 & 0.831 & 3.003 \\
\hline Feature match & 19.040 & 35.524 & 8.971 & 48.518 & 30.590 & 43. & 0.000 & 49.285 & 27.443 & 41.263 & 11.028 & 47.818 \\
\hline Interlocking polygons & 11.691 & 25.903 & 2.695 & 34.370 & 17. & & 0.000 & 26.527 & 22.335 & 20.916 & 7.757 & 23.353 \\
\hline Spatial planning & 0.357 & 2.593 & 0.028 & 3.046 & 0.652 & & 0.000 & 2.466 & 0.751 & 2.372 & 0.240 & 2.466 \\
\hline Token search & 0.956 & 2.015 & 0.046 & & 2043 & & 0.000 & 2.167 & 1.859 & 2.156 & 0.587 & 1.927 \\
\hline Spatial rotations & 11.381 & 39.601 & 5.561 & & 21.031 & 366 & 0.000 & 46.959 & 22.505 & 39.065 & 6.985 & 45.483 \\
\hline
\end{tabular}

Actually, we have performed a much wider statistical analysis than reported here. In fact for each indicator, and for each group, we have also considered three different statistical tests, i.e., Friedman, Aligned Eriedman, and Quade (Derrac et al., 2011). Unfortunately, the paper would become too lengthy and would contain too many tables if we showed all of them here. In the following, we report a short summary of the results obtained in this way:

- Pearson for Group 1: for all the three statistical tests the best-performing algorithm turns out to be the SimpleLinear;

- Pearson for Group 2: all the three statistical tests conclude that the best-performing algorithm is IBk;

- MAE for Group 1: there are two algorithms that are statistically better, namely REPTree and SimpleLinear;

- MAE for Group 2: all the three statistical tests conclude that the best-performing algorithm is REPTree;

- RMSE for Group 1: SimpleLinear is statistically better for all the three statistical tests;
- RMSE for Group 2: all the three statistical tests conclude that the best-performing algorithm is REPTree.

\section{DISCUSSION}

The importance of exercise to our overall well-being is welldocumented in the scientific literature. It is recommended in the prevention and management of various medical conditions, including cardiovascular disease, metabolic syndrome, osteoporosis, and so on. Additionally, the benefits of exercise extend beyond physiological changes. Exercise also benefits mental health, having been shown to improve self-esteem and self-confidence and to reduce symptoms of depression.

However, an often-overlooked aspect of exercise is the effect it has on cognition and the brain. Additionally, as far as reported in the literature, none of the published studies gives the possibility to predict the cognitive performance of the subjects. This possibility is what we investigate in this paper, and represents its novelty.

We have investigated the impact of physical activity on the cognitive performances measured by using a number of different 
TABLE 5 | Numerical results, obtained over train and test sets, related to RMSE.

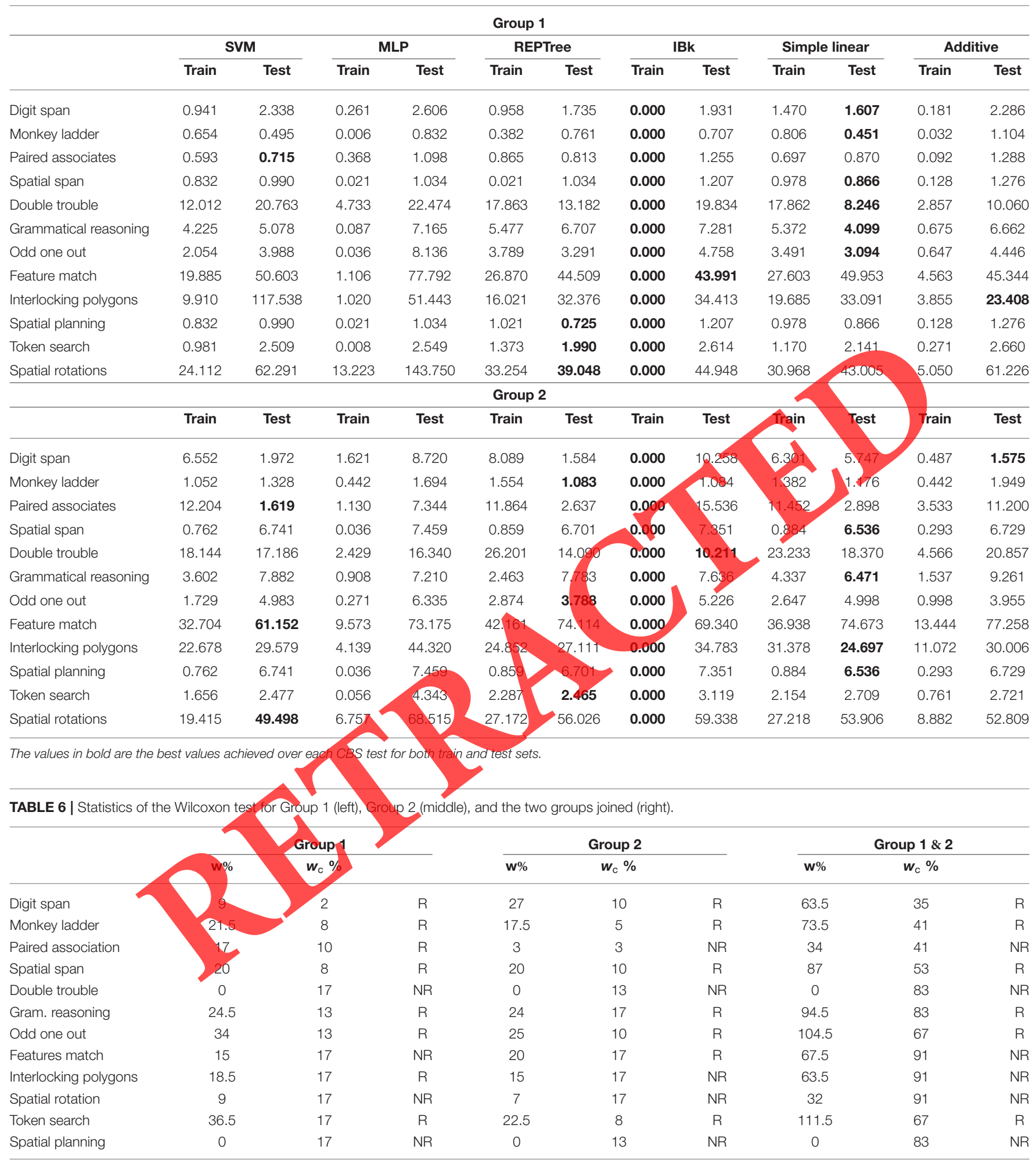

cognitive tests taken from CBS. To do this, we have chosen to use the HRV, because the dynamic autonomic responses during exercise can be measured by analyzing the ECG signal, i.e., the
HRV. In particular, we were interested in knowing whether HRV and variations in HRV parameters are associated with changes in the cognitive domain, that is, how the physical activity, measured 
TABLE 7 | The results of the Quade test for the three indicators for the subjects in Group 1.

\begin{tabular}{ccccccc}
\hline & Simple linear & REPTree & Additive & SVM & IBk & MLP \\
\hline Score & 2.098 & 2.955 & 3.353 & 3.800 & 4.048 & \\
& Statistic: 11.598 & & $\boldsymbol{p}$-value: 0.000 & & &
\end{tabular}

TABLE 8 | Post-hoc procedure for Quade test over Group 1 subjects.

\begin{tabular}{cccccccc}
\hline Algorithm & $\mathbf{p}$ & $\mathbf{z}$ & Bonf. & Holm & Holl. & Rom & Finner \\
\hline MLP & 0.001 & 3.312 & 0.001 & 0.010 & 0.010 & 0.011 & 0.010 \\
IBk & 0.015 & 2.439 & 0.015 & 0.013 & 0.013 & 0.013 & 0.020 \\
SVM & 0.033 & 2.129 & 0.033 & 0.017 & 0.017 & 0.017 & 0.030 \\
Additive & 0.116 & 1.570 & 0.116 & 0.025 & 0.025 & 0.025 & 0.038 \\
REPTree & 0.284 & 1.072 & 0.284 & 0.050 & 0.050 & 0.050 & 0.038 \\
Th & & & 0.010 & 0.013 & 0.013 & 0.011 & 0.030 \\
\hline
\end{tabular}

TABLE 9 | The results of the Quade test for the three indicators for the subjects in Group 2.

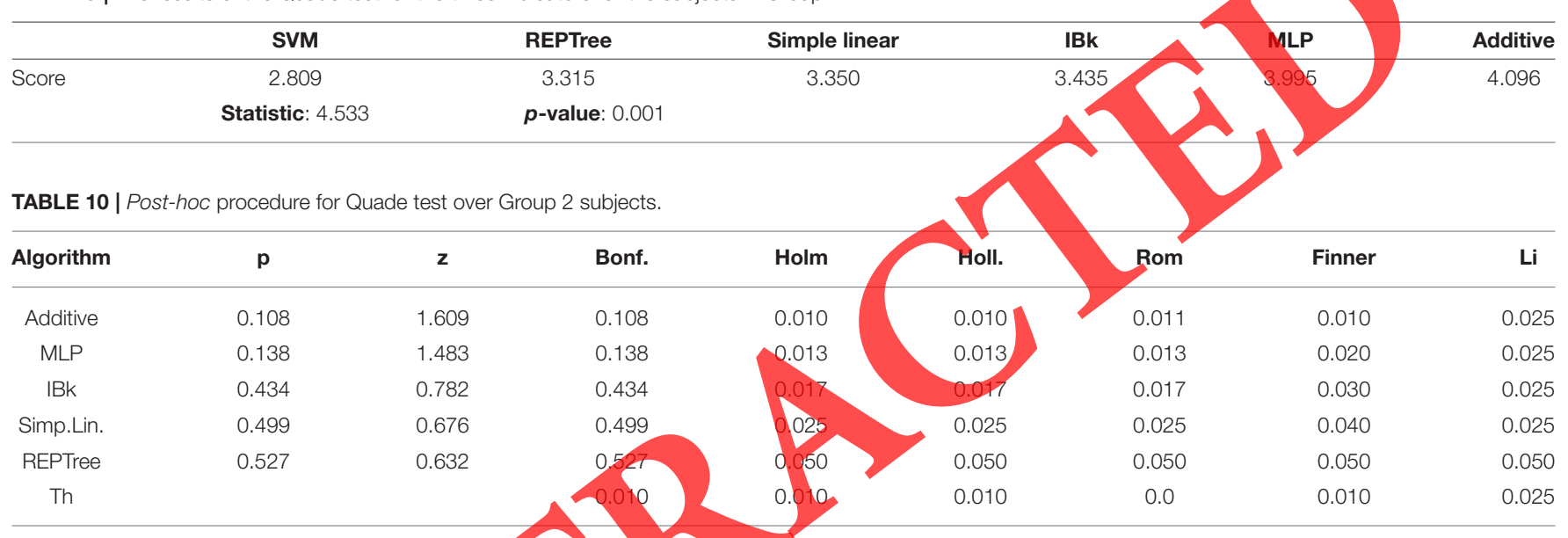

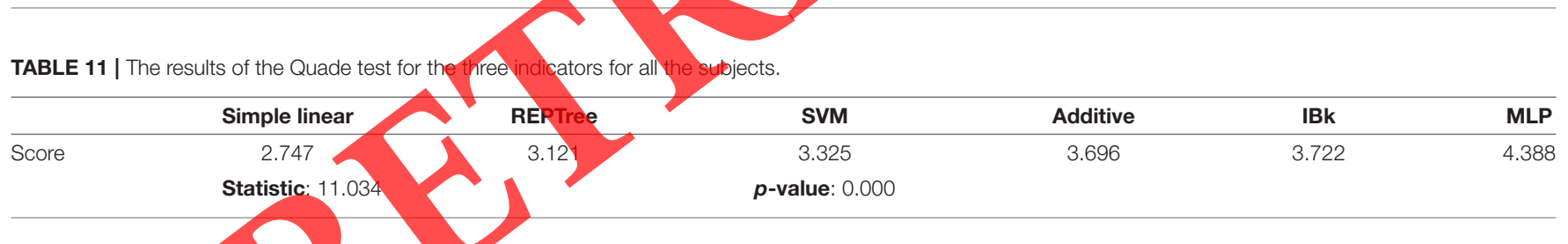

\begin{tabular}{|c|c|c|c|c|c|c|c|c|}
\hline IBK & 0.086 & 1.717 & 0.086 & 0.013 & 0.013 & 0.013 & 0.020 & 0.026 \\
\hline Additive & 0.094 & 1.673 & 0.094 & 0.017 & 0.017 & 0.017 & 0.030 & 0.026 \\
\hline SVM & 0.308 & 1.019 & 0.308 & 0.025 & 0.025 & 0.025 & 0.040 & 0.026 \\
\hline Th & & & 0.010 & 0.013 & 0.013 & 0.011 & 0.020 & 0.026 \\
\hline
\end{tabular}

by the variation in HRV, impacts on cognitive activities such as reasoning, work memory, and verbal skills.

We have performed the first analysis to determine whether the different exercise regimes (aerobic vs. effort exercise) had different effects on cognition. It has demonstrated that in most
CBS tests there is an improvement, or at least a confirmation, of the subject's cognitive ability, both for effort workout (group 1) and for aerobic one (group 2). In particular, reasoning, concentration, and planning tests seem to undergo important positive changes due to physical exercise. 
Secondly, a prediction analysis has been made in order to understand if, by monitoring the HRV during the exercise, it is possible to predict the cognitive performance, i.e., the CBS tests results. The regression analysis has demonstrated that this is possible to some extent, in terms of $\rho$, MAE, and RMSE. The results show that the best regression algorithm able to predict the cognitive performance by using the HRV is Simple Linear, or, subordinately, REPTre. Going into details, the best regression quality, in terms of $\rho$, MAE and RMSE, has been reached by a simple algorithm.

Summarizing, this study had numerous strengths, including the use of a large cognitive test battery, which allowed us to examine the effects of exercise on a range of cognitive functions. We compared two distinct exercise regimes, one of which focused on resistance exercise. The effects of long-term resistance exercise regimes have not been studied in healthy adults, and thus these results are novel.

Additionally, as mentioned previously, no other studies have investigated the possibility of predicting cognitive abilities through a simple mathematical relationship expressed by Simple Linear. Our study suggests the possibility of using this model in a real-time mobile application that, monitoring HRV during exercise, could predict cognitive performance. If needed, this application could also suggest the subject to modify the physical training in order to improve the predicted cognitive performance. This app could be useful within cognitive rehabilitation, where only cognitive digital games are currently employed. In this way, to these latter an appmonitored personalized physical activity could be associated with maximizing cognitive improvements. Moreover, this app could also be applied to support active aging, so as to contribute to avoiding, or at least to slowing down, the onset of pathologies as, e.g., dementia, cognitive impairment, cognitive decline, and so on.

Despite these strengths, this study had limitations that should be addressed in the future. Among them, one is that no additional information, as for example the participant's diet, which is recognized in the literature as impacting mental health (Loughrey et al., 2017), was taken into consideration.

\section{REFERENCES}

Aha, D. W., Kibler, D., and Albert, M. K. (1991). Instance-based learning algorithms. Mach. Learn. 6, 37-66. doi: 10.1007/BF00153759

Ahlskog, J. E., Geda, Y. E., Graff-Radford, N. R., and Petersen, R. C. (2011). "Physical exercise as a preventive or disease-modifying treatment of dementia and brain aging," in Mayo Clinic Proceedings (Rochester, Minnesota: Elsevier), 876-884. doi: 10.4065/mcp.2011.0252

Bauman, A., Merom, D., Bull, F. C., Buchner, D. M., and Fiatarone Singh, M. A. (2016). Updating the evidence for physical activity: summative reviews of the epidemiological evidence, prevalence, and interventions to promote “active aging." Gerontologist 56(Suppl_2):S268-S280. doi: 10.1093/geront/ gnw031

Bherer, L. (2015). Cognitive plasticity in older adults: effects of cognitive training and physical exercise. Ann. N. Y. Acad. Sci. 1337, 1-6. doi: 10.1111/nyas.12682

Blondell, S. J., Hammersley-Mather, R., and Veerman, J. L. (2014). Does physical activity prevent cognitive decline and dementia?: A systematic review
Additionally, the design of the study allowed us to examine the associations between cognition improvements and physical activity, but it did not allow us to establish the temporality of the observed relationships. As a consequence, we do not know if the effects of physical activity on cognition are short-term or last longer.

Finally, unfortunately, there is no guarantee about the applicability of our results, based on a sample of young and middle-aged adults, to older individuals. However, the results presented here are to be considered preliminary and could be used as a starting point for a new and more numerous pilot study. In fact, we plan to create a wider data set by enrolling more subjects representative of the population, and to make it available to the scientific community. This would allow us to create a freely available database on this specific problem.

\section{DATA AVAILABILITY STATEMENT}

The datasets used and/or analysed during the current study are available from the corresponding author on reasonable request.

\section{ETHICS STATEMENT}

The studies involving human participants were reviewed and approved by CNR Ethics Committee. The patients/participants provided their written informed consent to participate in

\section{AUTHOR CONTRIBUTIONS}

All authors contributed to the conception and the design of this study. Material preparation and data collection were performed by GS. Instead, the analysis was carried out by GS and ID. The first draft of the manuscript was written by GS and ID. All authors commented on the previous versions of this manuscript and read and approved the final manuscript.

and meta-analysis of longitudinal studies. BMC Public Health 14, 1-12. doi: 10.1186/1471-2458-14-510

Booth, F. W., and Lees, S. J. (2006). Physically active subjects should be the control group. Med. Sci. Sports Exerc. 38, 405-406. doi: 10.1249/01.mss.0000205117.11882.65

Bouguila, N., and Amayri, O. (2009). A discrete mixture-based kernel for SVMs: application to spam and image categorization. Inf. Process. Manage. 45, 631-642. doi: 10.1016/j.ipm.2009.05.005

Breslow, L. A., and Aha, D. W. (1997). Simplifying decision trees: a survey. Knowledge Eng. Rev. 12, 1-40. doi: 10.1017/S026988899700 0015

Chan, A.-W., Tetzlaff, J. M., Gøtzsche, P. C., Altman, D. G., Mann, H., Berlin, J. A., et al. (2013). Spirit 2013 explanation and elaboration: guidance for protocols of clinical trials. Brit. Med. J. 346, 1-42. doi: 10.1136/bmj.e7586

Cotman, C. W., Berchtold, N. C., and Christie, L.-A. (2007). Exercise builds brain health: key roles of growth factor cascades and inflammation. Trends Neurosci. 30, 464-472. doi: 10.1016/j.tins.2007.06.011 
Daskalov, I., and Christov, I. (1997). Improvement of resolution in measurement of electrocardiogram rr intervals by interpolation. Med. Eng. Phys. 19, 375-379. doi: 10.1016/S1350-4533(96)00067-7

Demsar, J. (2006). Statistical comparisons of classifiers over multiple data sets. J. Mach. Learn. Res. 7, 1-30.

Derrac, J., Garcia, S., Molina, D., and Herrera, F. (2011). A practical tutorial on the use of nonparametric statistical tests as a methodology for comparing evolutionary and swarm intelligence algorithms. Swarm Evol. Comput. 1, 3-18. doi: 10.1016/j.swevo.2011.02.002

Erickson, K. I., Voss, M. W., Prakash, R. S., Basak, C., Szabo, A., Chaddock, L., et al. (2011). Exercise training increases size of hippocampus and improves memory. Proc. Natl. Acad. Sci. U.S.A. 108, 3017-3022. doi: 10.1073/pnas.1015950108

Fletcher, G. F., Blair, S. N., Blumenthal, J., Caspersen, C., Chaitman, B., Epstein, S., et al. (1992). Statement on exercise: benefits and recommendations for physical activity programs for all Americans-a statement for health professionals by the committee on exercise and cardiac rehabilitation of the council on clinical cardiology, American Heart Association. Circulation 86:340. doi: 10.1161/01.CIR.86.1.340

Garner, S. R. (1995). "WEKA: the Waikato environment for knowledge analysis," in Proceedings of the New Zealand Computer Science Research Students Conference, 57-64.

Hampshire, A., Highfield, R. R., Parkin, B. L., and Owen, A. M. (2012). Fractionating human intelligence. Neuron 76, 1225-1237. doi: 10.1016/j.neuron.2012.06.022

Lange, K. W., Robbins, T., Marsden, C., James, M., Owen, A., and Paul, G. (1992). L-dopa withdrawal in Parkinson's disease selectively impairs cognitive performance in tests sensitive to frontal lobe dysfunction. Psychopharmacology 107, 394-404. doi: 10.1007/BF02245167

Loughrey, D. G., Lavecchia, S., Brennan, S., Lawlor, B. A., and Kelly, M. E. (2017). The impact of the mediterranean diet on the cognitive functioning of healthy older adults: a systematic review and meta-analysis. Adv. Nutr. 8, 571-586. doi: 10.3945/an.117.015495

Luft, C. D. B., Takase, E., and Darby, D. (2009). Heart rate variability and cognitive function: effects of physical effort. Biol. Psychol. 82, 186-191 doi: 10.1016/j.biopsycho.2009.07.007

Luque-Casado, A., Zabala, M., Morales, E., Mateo-March, M., and Sanabria, D. (2013). Cognitive performance and heart rate variability: the influence of fitness level. PLoS ONE 8:e56935. doi: 10.1371/journal.pone.0056935

Makivić, B., Nikić Djordjević, M., and Willis, M. S. (2013) physical activities. J. Exerc. Physiol. 16, 103-131.

Mateo, J., and Laguna, P. (2000). Improved heart rate variability signal analysis from the beat occurrence times according to the IPFM model. IEEE Trans. Biomed. Eng. 47, 985-996. doi: 10.1109/10.855925

Mehta, M. A., Owen, A. M., Sahakian, B. J., Mavaddat, N, Pickard, J. D., and Robbins, T. W. (2000). Methylphenidate enhances working memory by modulating discrete frontal and parietal lobe regions in the human brain. $J$. Neurosci. 20, RC65. dor: 10.1523/JNEURQSC1.20-06-j0004.2000

Ngandu, T., Lehtisalo, J., Solomon, A, Levälahti, E., Ahtiluoto, S., Antikainen, R., et al. (2015). A 2 year multidomain intervention of diet, exercise, cognitive training, and vascular risk monitoring versus control to prevent cognitive decline in at-risk elderly people (finger): a randomised controlled trial. Lancet 385, 2255-2263. doi: 10.1016/S0140-6736(15)60461-5

Owen, A., James, M., Leigh, P., Summers, B., Marsden, C., Quinn, N. P., et al. (1992). Fronto-striatal cognitive deficits at different stages of Parkinson's disease. Brain 115, 1727-1751. doi: 10.1093/brain/115.6.1727

Owen, A. M., Beksinska, M., James, M., Leigh, P., Summers, B., Marsden, C., et al. (1993). Visuospatial memory deficits at different stages of Parkinson's disease. Neuropsychologia 31, 627. doi: 10.1016/0028-3932(93)90135-M

Owen, A. M., Downes, J. J., Sahakian, B. J., Polkey, C. E., and Robbins, T. W. (1990). Planning and spatial working memory following frontal lobe lesions in man. Neuropsychologia 28, 1021-1034. doi: 10.1016/0028-3932(90) 90137-D

Owen, A. M., Doyon, J., Petrides, M., and Evans, A. C. (1996a). Planning and spatial working memory: a positron emission tomography study in humans. Eur. J. Neurosci. 8, 353-364. doi: 10.1111/j.1460-9568.1996.tb 01219.x

Owen, A. M., Evans, A. C., and Petrides, M. (1996b). Evidence for a twostage model of spatial working memory processing within the lateral frontal cortex: a positron emission tomography study. Cereb. Cortex 6, 31-38. doi: $10.1093 /$ cercor/6.1.31

Owen, A. M., Roberts, A. C., Polkey, C. E., Sahakian, B. J., and Robbins, T. W. (1991). Extra-dimensional versus intra-dimensional set shifting performance following frontal lobe excisions, temporal lobe excisions or amygdalo-hippocampectomy in man. Neuropsychologia 29, 993-1006. doi: 10.1016/0028-3932(91)90063-E

Paterson, D. H., and Warburton, D. E. (2010). Physical activity and functional limitations in older adults: a systematic review related to Canada's physical activity guidelines. Int. J. Behav. Nutr. Phys. Act. 7:38. doi: 10.1186/1479-5868-7-38

Prakash, R. S., Voss, M. W., Erickson, K. I., and Kramer, A. F. (2015). Physical activity and cognitive vitality. Annu. Rev. Psychol. 66, 769-797. doi: 10.1146/annurev-psych-010814-015249

Rumelhart, D. E., Hinton, G. E., Williams, R. J. (1988). Learning representations by back-propagating errors. Cogn. Model. 5, 533-536.

Scherder, E., Scherder, R., Verburgh, L., Königs, M., Blom, M., Kramer, A. F., et al. (2014). Executive functions of sedentary elderly may benefit from walking: a systematic review and meta-analysis, Am. Y. Geriatr. Psychiatry 22, 782-791. doi: 10.1016/j.jagp.2012.12.026

Sharp, N. C. (1993). Guidelines for exercise testing and prescription. Brit. J. Sports Med. 27:138. doi: 10.1136/

Stone, C. J. (1985). Additive regression and other nonparametric models. Ann. Stat. 13, 689-705. doi. 10.1214/aos/11763

Tarvainen, M. P., Niskanen, J.-P., Lipponen, J. A., Ranta-Aho, P. O., and Karjalainen, P. A. (2014). Kubios HRV-heart rate variability analysis software. Comput. Methods Programs Biomed. 113, 210-220. do: 10.1016/j.cmpb.2013.07.024

Tivadar, B. K. (2017). Physical activity improves cognition: possible explanations. Biogerontology 18, 477-483. doi: 10.1007/s10522-0179708-6

Tsunoda, K., Chiba, A., Chigira, H., Ura, T., and Mizunq, O. (2015). “Estimating changes in a cognitive performance using heart rate variability," in 15th IEEE International Conference on Bioinformatics and Bioengineering (BIBE) (Belgrade: IEEE), 1-6. doi: 10.1109/BIBE.2015.7367712

Vaynman, S., and Gomez-Pinilla, F. (2006). Revenge of the "sit": how lifestyle impacts neuronal and cognitive health through molecular systems that interface energy metabolism with neuronal plasticity. J. Neurosci. Res. 84, 699-715. doi: 10.1002/jnr.20979

World Medical Association (2008). Declaration of Helsinki. Ethical Principles for Medical Research Involving Human Subjects. Available online at: https://www. wma.net/policies-post/wma-declaration-of-helsinki-ethical-principles-formedical-research-involving-human-subjects/

Zeng, Z.-Q., Yu, H.-B., Xu, H.-R., Xie, Y.-Q., and Gao, J. (2008). "Fast training support vector machines using parallel sequential minimal optimization," in 3 rd International Conference on Intelligent System and Knowledge Engineering (Xiamen: IEEE), 997-1001.

Zephyr Technology (2016). New Zephyr BioPatch Monitoring Device for Human Performance.

Conflict of Interest: The authors declare that the research was conducted in the absence of any commercial or financial relationships that could be construed as a potential conflict of interest.

Copyright (C) 2020 Sannino, De Falco, De Pietro and Stranges. This is an open-access article distributed under the terms of the Creative Commons Attribution License (CC $B Y)$. The use, distribution or reproduction in other forums is permitted, provided the original author(s) and the copyright owner(s) are credited and that the original publication in this journal is cited, in accordance with accepted academic practice. No use, distribution or reproduction is permitted which does not comply with these terms. 